\title{
La modélisation en assainissement et en environnement : une synthèse des préoccupations des acteurs de l'environnement
}

\author{
Urban Drainage and Environmental Modelling : \\ A Synthesis of the Environment Actor's Preoccupations
}

par L. Phan

Anjou Recherche

J-M. Brignon

Compagnie Générale des Eaux

Since the beginning, the hydraulical modelling applied to the environment never stopped its evolution from the computation of flow in the sewer networks and the rivers to the propagation and evolution of the pollution within those systems. On the same time, these models turned from research objects to largelly used engineering tools, with heavy consequences on their further developments. Thus, it is important to maintain the link between research and innovation, on one side, large use and industrialisation, on the other side.

\section{I — INTRODUCTION}

En vue de l'étude de l'environnement et de l'impact que peut avoir sur celui-ci l'assainissement des zones urbaines, la modélisation des phénomènes hydrauliques puis de pollution s'est développée depuis plusieurs années, tant sur le système d'assainissement urbain, que pour les études fluviales.

Les progrès scientifiques et techniques, mais aussi l'évolution de la demande des ingénieurs et des maîtres d'ouvrage (pour la plupart des services publics, des collectivités locales et les populations qu'ils représentent) ont cependant radicalement transformé le milieu de la modélisation.

\section{U UN DÉVELOPPEMENT TECHNIQUE CONTINU}

La modélisation en hydraulique trouve sa première expression dans les modèles réduits utilisés dès les années 1930 . Appliqués en hydraulique à surface libre, en particulier pour des problèmes de dimensionnement de canaux, ils sont tout d'abord cantonnés à un rôle d'illustration. Leur utilisation intensive pour le dimensionnement d'ouvrages hydrauliques, et la prise de décision vient rapidement ensuite. Mais, après quelques décennies d'un " âge d'or ", la difficulté d'étudier ainsi les systèmes de très grande taille (bassins versants, grands estuaires) et le coût élevé de ces maquettes, parallèlement avec les perfectionnements rapides de l'informatique, favorisent l'essor de la modélisation mathématique sur ordinateur.
Les premiers modèles sont utilisés pour le dimensionnement des réseaux en vue de résoudre les problèmes de débordement, ainsi qu'en modélisation hydraulique fluviale pour la construction de canaux et d'aménagements des fleuves. Les enjeux sont donc, à cette époque, toujours limités à I'horizon hydraulique. Par conséquent, les liens entre les modèles de réseaux et les modèles d'hydraulique à surface libre sont faibles : les actions hydrauliques réciproques entre des réseaux et des rivières sont en général limitées.

Puis, avec les deux versions successives de la loi sur l'eau (1964 et 1992), les préoccupations pour la qualité de l'eau, en particulier l'impact des rejets d'eaux usées sur la qualité des rivières, s'affirment. L'article 10 de la loi du 3 janvier 1992, et le décret d'application $93742 / 743$ du 29 mars 1993, renforcent la notion d'un continuum entre réseaux et cours d'eau. Dans ce cadre, on voit deux tendances nouvelles en modélisation émerger puis prendre véritablement le devant de la scène ces dernières années :

- Les modèles se dotent de " modules qualité " de plus en plus sophistiqués, configurables, traitant progressivement de tous les aspects de la pollution (dissoute, particulaire,...) et de tous les paramètres (pollutions organiques, azotées, métaux lourds, aspects piscicoles,...).

- Le lien entre modèles de réseaux et les modèles de rivière s'accentuent : il doit être possible de les utiliser " en chaîne ", des réseaux à l'estuaire en passant par les rivières. Ils doivent donc être capables d'échanger des informations, et si possible faire partie d'un même environnement logiciel $|1,5|$. 
Cette évolution technique vers une plus grande sophistication et une plus grande intégration des modèles numériques, s'est dès le départ doublée d'une évolution que l'on pourrait qualifier de "sociale", qui a vu passer la modélisation numérique du stade d'instrument de recherche à celui d'outil de calcul et de démonstration largement répandu.

Loin d'être un facteur secondaire, portant sur la façon " d'habiller " les logiciels, la diffusion de ces outils, du cercle étroit des chercheurs vers un plus large public d'ingénieurs et techniciens, puis de décideurs, chacun avec ses propres objectifs et préoccupations, va avoir une importance capitale quant au développement de la modélisation et des recherches associées.

\section{III $\square$ PREMIER STADE : LES MODÈLES DE RECHERCHE}

Les modèles, qu'ils soient d'assainissement ou fluviaux, sont tout naturellement issus de la recherche scientifique pour laquelle ils constituent des outils de compréhension des phénomènes physiques et biochimiques, ainsi que de validation des hypothèses à prendre en compte dans les études et recherches dans le domaine de l'environnement.

Ce domaine se caractérise par la multiplicité desdits phénomènes ainsi que leurs relations d'interdépendance, le tout se basant de près ou de loin sur l'hydraulique, qui en reste le support central (voir figure 1).
Ces phénomènes eux-mêmes peuvent être traités ou retraduits de multiples manières, correspondant non seulement aux hypothèses de travail des différentes équipes de chercheurs, mais aussi à la place qu'ils occupent dans leurs préoccupations [3].

Typiquement, un modèle, qu'il soit d'assainissement ou de rivière, pourra différer d'un autre, devant traiter du même problème, par :

- les équations utilisées pour simuler chaque phénomène ; exemple : pour le transport solide, modèles de Wiuff, Ackers-White, Engelund-Hansen...[4],

- les hypothèses simplificatrices adoptées pour la résolution des mêmes équations de base ; en hydraulique, par exemple, la résolution des équations complètes de Barré-de-SaintVenant est souvent remplacée par celle des équations de l'onde cinématique, ou diffusante, plus simples à traiter, mais au domaine de validité (pour obtenir un résultat final correct) réduit [5],

- le schéma de résolution numérique utilisé, qui influe directement sur la robustesse, la rapidité et la précision du modèle,

- l'interface homme-machine, mais ceci est accessoire.

Il ne saurait être question d'émettre un jugement de valeur dans ce contexte, sauf sur de stricts aspects fonctionnels (et accessoires), sur les programmes développés.

Ces modèles ont été conçus et développés en fonction des besoins et orientations de leurs utilisateurs, qui, pour des rai-

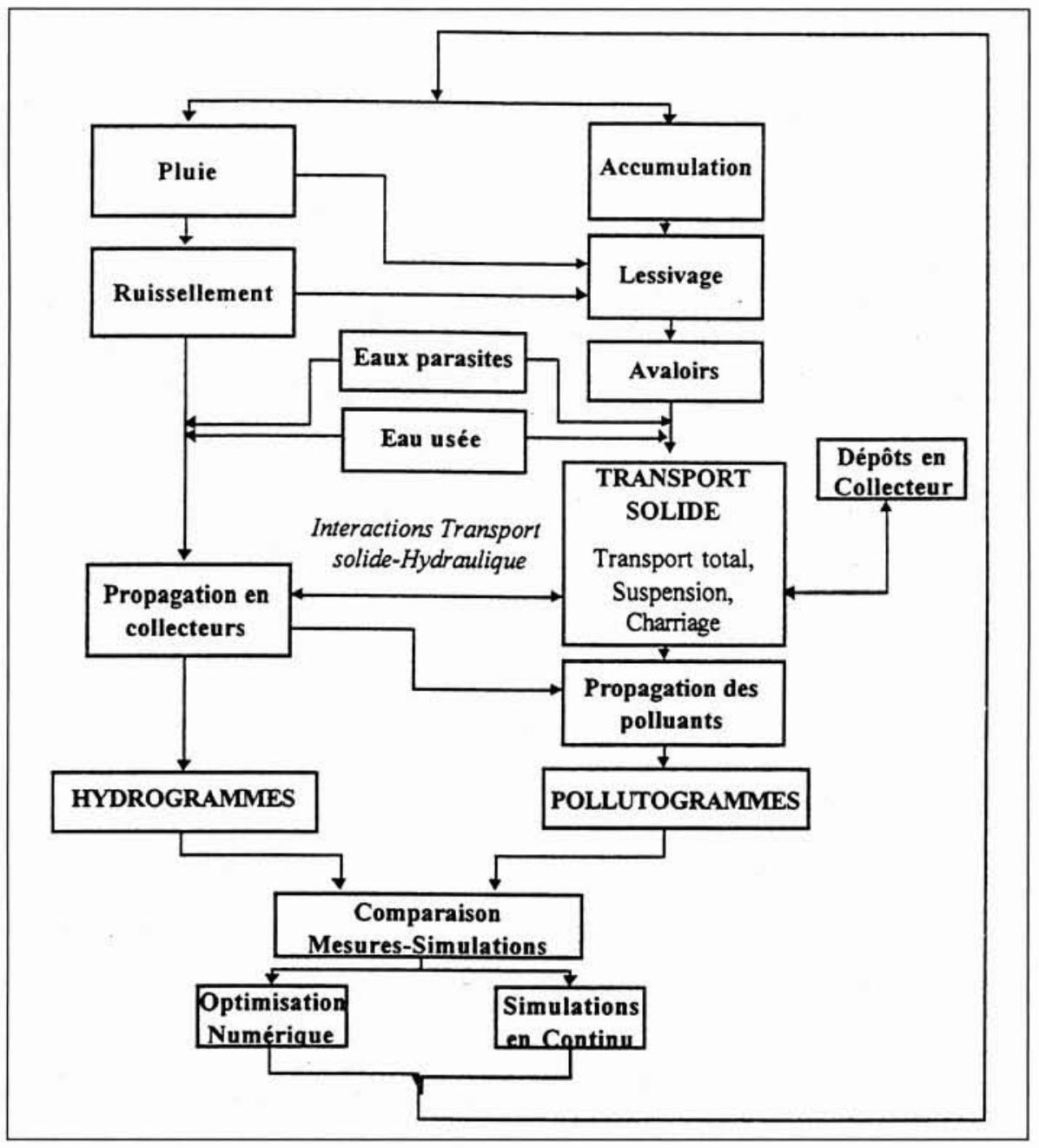

1. Schéma d'architecture d'Horus - Structure d'un programme de modélisation AssainissementPollution (tiré de [2]). 
sons éminemment pratiques, peuvent choisir une méthode simplifiée, voire rudimentaire, pour le traitement d'un phénomène connexe à leur sujet principal.

L'étude d'une usine de dépollution peut, par exemple, se focaliser sur le procédé biochimique, en considérant tous les problèmes hydrauliques comme résolus (mélanges parfaits, stabilité et équirépartition des débits dans les ouvrages...), ce qui dans la réalité est loin d'être toujours le cas !

La recherche a ainsi fourni - et continuera de fournir - une grande variété de modèles, qui ne sauraient généralement être comparés entre eux, utilisés et adaptés par des spécialistes maîtrisant leurs hypothèses de travail et conditions d'emploi.

Pour de tels outils et un tel usage à moyen et long termes, les aspects convivialité et performances informatiques (rapidité) des programmes, s'ils sont appréciés, ne sont cependant pas capitaux.

\section{IV — DEUXIÈME STADE : APPLICATION À L'INGÉNIERIE}

Un grand nombre de logiciels de modélisation en particulier en France, ont été développés et sont encore utilisés au sein des bureaux d'études ou des centres techniques publics, en hydraulique en premier lieu, plus rarement et tardivement en pollution. Ce dernier décalage correspond à celui de la demande, mais est également consécutif à la complexité du traitement de la pollution, les problèmes posés étant généralement pluridisciplinaires.

Les ingénieurs et techniciens en charge des études d'environnement et d'aménagement ont commencé par appliquer les algorithmes et formules, quand ce n'étaient pas directement les outils, issus de la recherche, sélectionnant et adaptant ceux-ci en fonction des problèmes qu'ils avaient à résoudre.

Ces modèles à usage d'étude, présentent ainsi une grande diversité, qui reflète celle des outils de recherche dont ils sont issus, mais aussi les contraintes matérielles (informatiques) prévalant au moment de leur élaboration et les objectifs fixés en fonction des besoins spécifiques rencontrés sur le marché.

Par exemple, dans le but de veiller à la conformité des systèmes d'assainissement quant à des objectifs normalisés de limitation des rejets polluants au milieu naturel, certaines équipes (plus particulièrement du nord de l'Europe, où un grand effort de mesure systématique des rejets polluants a été entrepris de longue date) se sont tournées vers l'usage de modèles de type stochastique, avec une hydraulique réduite à sa plus simple expression. L'objectif est ici, non plus de reproduire un événement physique, avec le calcul d'une courbe de pollution, mais d'évaluer des quantités globales de polluants rejetés sur de longues périodes. On s'éloigne ainsi du domaine de l'hydraulique pour une application de techniques statistiques.

Les modèles hydrologiques urbains, les modèles de transformation pluie-débit, sont une autre illustration de la diversité des techniques en usage. A partir des travaux de recherche menés dans le domaine, un certain nombre de modèles " nationaux " se sont dégagés pour devenir les plus couramment utilisés dans leurs pays respectifs, sans nécessairement que cela soit de façon exclusive. Si la France utilise ainsi le modèle du Réservoir Linéaire [6], le Royaume-Uni peut employer la Procédure mise au point à Wallingford (Angleterre) et l'Allemagne le modèle Hystem.
Plus que les modèles utilisés, c'est la manière de les mettre en cuvre et d'exploiter leurs calculs, laquelle reflète le savoir-faire des équipes d'étude, qui fait la différence. Une étude peut être menée avec un logiciel de calcul moins évolué hydrauliquement sans pour autant que sa validité soit remise en cause du fait de l'existence d'outils plus sophistiqués.

Comme pour les programmes de recherche, une comparaison paraît donc difficile à mettre en place pour la plupart de ces logiciels de modélisation en raison de la disparité des objectifs comme des conditions de mise en œuvre et d'exploitation.

Cette situation va changer avec l'apparition de logiciels commerciaux qui va de pair avec une implication plus directe des maîtres d'ouvrage dans les études.

\section{V $\square$ STADE ACTUEL : LES OUTILS INDUSTRIELS}

Les modèles d'étude dont il a été question plus haut étaient pour la plupart des outils développés au sein d'unités d'ingénierie, principalement à des fins d'usage interne. Ces développements étaient donc le fait d'équipes le plus souvent de taille réduite, proches des utilisateurs (qui sont parfois les mêmes) et combinant ingénierie et informatique.

De tels outils, même s'ils sont de grande qualité, ont de plus en plus tendance à céder la place à des logiciels développés par des sociétés spécialisées. Les raisons de cette évolution sont multiples :

- l'extension des études ayant recours à la modélisation a eu pour conséquence un élargissement de l'usage des modèles au-delà du cercle des experts et des ingénieurs spécialisés. Cette " démocratisation" des modèles a appelé au développement d'une convivialité plus soignée et de performances de calcul plus élevées (rapidité et précision), la plupart des utilisateurs ne pouvant se permettre ni un long apprentissage ni une mise en œuvre trop longue du fait d'une demande de productivité accrue.

- dans le même esprit, maintenance, mises à jour (pour suivre les progrès techniques) et support technique (pour résoudre les problèmes que peuvent rencontrer les utilisateurs) des logiciels nécessitent dorénavant I'intervention d'équipes spécialisées et disponibles, donc spécifiquement dédiées à cette tâche.

- enfin, au-delà de la remise de résultats d'études, la demande d'information et d'explication des maîtres d'ouvrage, principalement les collectivités, s'est faite plus importante. Il y a été répondu par le développement de fonctions permettant une présentation plus synthétique et aisément appréhendable des résultats.

La conséquence de ces différents facteurs est l'accroissement de la charge de développement et de services dédiés, une charge que les petites équipes d'ingénieurs d'étude ne peuvent généralement plus assumer. La conclusion en est : " à usage industriel, outil industriel ".

De nouveaux modèles commerciaux sont donc diffusés aujourd'hui avec comme caractéristiques communes :

- une " fermeture " du programme pour d'évidentes raisons commerciales et de concurrence,

- une convivialité soignée,

- la recherche de bonnes performances de calcul, en précision, stabilité et rapidité, 
- une tendance à traiter des phénomènes de la manière la plus extensive possible, afin de limiter les simplifications et hypothèses réductrices qui seraient du fait du modèle et permettre à l'utilisateur de traiter la plus grande variété de cas possibles.

Une certaine standardisation, fonction des besoins les plus couramment exprimés a donc eu lieu et pour un objectif d'étude bien défini, plusieurs modèles standardisés peuvent désormais se trouver en concurrence et être comparés. L'industrialisation, en séparant équipes d'étude et équipes de développement a ainsi établi la distinction entre performance d'un modèle et savoir-faire de l'utilisateur.

Cet état de fait a cependant l'inconvénient de rendre les chargés d'étude dépendants des possibilités et performances des outils à leur disposition, sans pouvoir adapter directement ceux-ci à des cas ou conditions inédits qui peuvent se rencontrer, notamment à l'export. On peut ainsi penser à l'hydrologie tropicale, si différente des conditions prévalant en zone tempérée, où ont été mis au point une grande partie des modèles (mais il n'est pas toujours nécessaire d'aller chercher aussi loin le contre-exemple).

Le chargé d'étude, ne pouvant résoudre directement le problème, sera amené à utiliser des palliatifs comme l'utilisation sous hypothèse d'une fonction existante de son modèle ou à renoncer à l'utilisation de celui-ci au profit d'un calcul particulier.

L'adaptation des logiciels de modélisation à de nouveaux problèmes ou de nouveaux cas de figure dépend donc désormais de la capacité de prise en compte de ceux-ci par les équipes de développement. Ces dernières cherchant à regrouper et utiliser les multiples compétences nécessaires à une prise en compte correcte des phénomènes, des groupements internationaux (principalement européens et souvent avec participation française) sont apparus autour des grands logiciels commerciaux pour en assurer le développement et la validation.

Ces regroupements ne sauraient cependant à eux seuls garantir une évolution à la pointe du progrès et des connaissances, du fait des contraintes économiques et techniques qui pèsent sur le développement des modèles (en recherche, il est difficile et coûteux de remettre en question une option technique ; cela est encore plus vrai pour des produits commerciaux). Seule une recherche dynamique, capable d'explorer de nouvelles solutions et de les faire valider sur le terrain pourrait le faire, à condition de trouver les relais nécessaires auprès des organismes développeurs.

L'école française de l'eau, avec ses équipes de recherche et développement, ses ingénieurs et techniciens œuvrant au niveau mondial dans les organismes d'études ou au sein des collectivités locales, sera apte à remplir un tel rôle par le biais des partenariats nationaux ou internationaux qu'elle pourra nouer pour favoriser et accélérer le passage de la recherche à l'exploitation industrielle en ce domaine, tant il est vrai que le temps des pionniers individuels semble devoir être révolu.

\section{VI $\square$ CONCLUSION}

Les perspectives ouvertes par l'amélioration des performances et la généralisation de la micro-informatique sont multiples. Tout d'abord, un ample mouvement de démocratisation de l'utilisation des modèles est en cours. La plupart des décideurs souhaitent désormais adosser leur décision à l'emploi d'un modèle, si possible utilisé par leurs propres services. Cette démocratisation tend à faire baisser les coûts d'acquisition et d'utilisation des outils. Ceux-ci, sous la pression des utilisateurs, sont de plus en plus des outils intégrés, et de véritables produits logiciels " de grande consommation ". Le seul frein qui régule cette évolution réside dans la nécessité de disposer de données pour recourir à la modélisation, et au coût élevé de leur acquisition. Cependant, l'émergence de réseaux de mesures d'un coût d'investissement et de maintenance acceptables pourrait remettre en cause ce constat.

Cette transformation s'accomplit dans un cadre mondialisé : les pays en voie de développement, un peu à l'instar de ce qui se produit pour les techniques de communication (radiotéléphone, internet, satellite,...), souhaiteront probablement sauter les étapes intermédiaires pour directement s'intéresser aux outils intégrés " grand public " d'aide à la décision et à la gestion. Pour répondre à cette demande, de nouveaux processus accélérés de recherche / industrialisation de produits adaptés aux conditions hydrologiques et culturelles des pays émergents doivent être menés. C'est à cette condition que l'Ecole française de l'Eau disposera des outils de modélisation l'aidant à affirmer son rayonnement mondial.

\section{BIBLIOGRAPHIE}

[1] NIELSEN M, CARTENSEN J., HARREMOES P. 1996. Combined control of sewer treatment plant during rainstorm. Water Sciences and Technology, Vol. 34, $\mathrm{N}^{\circ} 3-4$.

[2] ZUG M. 1998. Modélisation conceptuelle de la pollution par temps de pluie en réseau d'assainissement. Thèse de doctorat de I'ULP, Strasbourg. $1998,241 \mathrm{p}$.

[3] PHAN L., HERREMANS L., DELAPLACE D, BLANC D, 1994 Modeling urban stormwater pollution : comparison between British (Mosqito) and French (Flupol) approaches. Conference of Hydroinformatics 94. Rotterdam, 1994, Vol. 1, pp 443-450.

[4] BERTRAND-KRAJEWSKI J.L., 1991. Modélisation des débits et du transport solide en réseau d'assainissement. Etude bibliographique. ENGEES, France.

[5] BUJON G. 1988. Prévision des débits et des flux polluants transités par les réseaux d'égouts par temps de pluie. Le modèle FLUPOL. La Houille Blanche, $N^{\circ} 1$, pp 11-23.

[6] DESBORDES M. 1984. Modélisation en hydrologie Urbaine Recherches et applications. LHM, 183 p. 